\title{
Fabrication and Characterization of Inhomogeneity-free Polymer Gels
}

\author{
M. Shibayama ${ }^{1}$, X. Li ${ }^{2}$, Y. Tsuji ${ }^{2}$, M. Ohira $^{2}$ \\ ${ }^{1}$ Neutron Science and Technology Center, Comprehensive Research Organization, Tokai, Ibaraki 319-1106, Japan \\ ${ }_{2}^{2}$ Institute for Solid State Physics, The University of Tokyo, Kashiwanoha, Kashiwa, Chiba, 277-8581, Japan \\ m_shibayama@cross.or.jp
}

Polymer gels are noncrystalline, disordered, and soft materials, consisting of infinite polymer networks and solvent. About 30 years have passed since small-angle neutron scattering (SANS) technique was applied to structure investigations of polymer gels, ${ }^{1}$ which was about 15 years later than its first applications to polymeric systems. ${ }^{2}$ Because of random cross-linking of the gel network, the structure of a gel is inhomogeneous over a wide spatial range from a few nanometers (the mesh size) to submicrons (large clusters). This is one of the reasons why scattering studies on gels started much later. Polymer gels are inherently much complicated systems than other polymeric systems. the gel network has various types of defects, entanglements, and a broad distribution of inter-crosslink chain length. As a result, structure investigations of polymer gels with SANS were far from quantitative level, even though SANS had been contributed to advances in polymer gel science, namely, deformation, ${ }^{3}$ swelling, polyelectrolytes, ${ }^{4}$ volume phase transition, ${ }^{5}$ etc. Though it was a dream for polymer chemists to fabricate "ideal" polymer networks, consisting of uniform mesh without defects, its realization had been unsuccessful. This lecture presents an overview of the history of fabrication and characterization of inhomogeneity-free polymer gels investigated by SANS, small-angle X-ray scattering, and light scattering.

A homogeneous gel consisting of monodisperse mesh size without negligible defects, named as tetra-PEG gel, was fabricated by cross-end-coupling of tetra-arm poly(ethylene glycol) (tetra-PEG) carrying complementary end groups. ${ }^{6}$ The network homogeneity was confirmed by SANS and by mechanical testing..$^{7-9}$ This methodology has brought a paradigm shift in synthesis, physics, and materials science of polymer gels, and various types of gels were prepared, e.g., ion gels, ${ }^{10}$ critical cluster gels, ${ }^{11} 12$

Owing to the development of gel fabrication, it is now possible to make a tailor-made multi-component polymer network gel with unimodal mesh size, such as $2 \times 4$ gels, ${ }^{13}$ and DNA-cross-linked physical gels. ${ }^{14}$ This has necessitated advancement of scattering theories for polymer gels. We have developed a scattering theory for multi-component polymer networks based on random phase approximation ${ }^{15} 16$ and applied it to structural analyses of DNA-cross-linked physical gels ${ }^{14}$ and deuterated/hydrogenous heteropolymer network gels ${ }^{17}$

[1] Baumgartner, A. Picot, C. E., Ed. (1989). Molecular Basis of Polymer Networks. 42 Berlin: Springer.

[2] Ballard, D. G. H. Schelten, J.. Wignall, G. D. (1973). Eur. Polym. J. 9, 965.

[3] Mendes, E. J. Lindner, P. Buzier, M. Boue, F. Bastide, J., (1991). Phys. Rev. Lett. 66, 1595.

[4] Moussaid, A. Schosseler, F. Munch, J. P. Candau, S. J. (1993). J. Phys. II France 3, 573.

[5] Shibayama, M. Tanaka, T. Han, C. C. (1992). J. Chem. Phys. 97, 6829.

[6] Sakai, T. Matsunaga, T. Yamamoto, Y. Ito, C. Yoshida, R. Suzuki, S. (2008). Macromolecules. 41, 5379.

[7] Matsunaga, T. Sakai, T. Akagi, Y. Chung, U. Shibayama, M. (2009). Macromolecules 42, 1344.

[8] Matsunaga, T. Sakai, T. Akagi, Y. Chung, U.. Shibayama, M. (2009). Macromolecules 42, 6245.

[9] Sakai, T. Akagi, Y. Matsunaga, T. Kurakazu, M. Chung, U. Shibayama, M. (2010). Macromolecular Rapid Communications. 31, 1954.

[10] Asai, H. Fujii, K. Ueki, T. Sakai, T. Chung, U. Watanabe, M. Han, Y. S. Kim, T. H. (2012). Macromolecules, 45, 3902.

[11] Li, X. Hirosawa, K. Sakai, T. Gilbert, E. P. Shibayama, M., (2017). Macromolecules 50, 3655.

[12] Hayashi, K. Okamoto, F. Hoshi, S. Katashima, T. Zujur, D. C.. Li, X. Shibayama, M. Gilbert, E. P. Chung, U. Ohba, S. Oshika, T. Sakai, T., (2017). Nat. Biomed. Eng. 1, 0044.

[13] Tsuji, Y. Li, X.. Shibayama, M., (2018) Gels, 4, 50.

[14] Li, X. Ohira, M. Naito, M. Shibayama, M. (2020). in preparation.

[15] Ijichi, Y. Hashimoto, T. (1988), Polym. Comm. 29, 135.

[16] Mortensen, K. Borger, A. L. Kirkensgaard, J. J. K. Garvey, C. J. Almdal, K. Dorokhin, A. Huang, Q. Hassager, O. (2018). Phys. Rev. Lett. 120, 207801.

[17] Ohira, M. Tsuji, Y. Watanabe, N. Morishima, K. Gilbert, E. P. Li, X.. Shibayama, M. (2020). Macromolecules $53,4047$.

\section{Keywords: polymer gels. tetra-PEG gel. small angle neutron scattering. random phase approximation}

